\title{
Research and Realization of a Medium Power Wireless Power Transmission System
}

\author{
Xiao Peng Li ${ }^{1,2}$, Xue Qing Yuan ${ }^{2, *}$, Kai Lin $\mathrm{Xu}^{2}$ \\ ${ }^{1}$ School of Computer and Control Engineering, University of the Chinese Academy of Sciences, Beijing, China \\ ${ }^{2}$ Shenyang Institute of Automation Chinese Academy of Sciences, State Key Laboratory of Robotics, Shenyang, China
}

\section{Email address:}

lxpxueshanfeitian@163.com (Xiao Peng Li), yuanxueqing@sia.cn (Xue Qing Yuan), xukailin@sia.cn (Kai Lin Xu)

${ }^{*}$ Corresponding author

\section{To cite this article:}

Xiao Peng Li, Xue Qing Yuan, Kai Lin Xu. Research and Realization of a Medium Power Wireless Power Transmission System. Science Discovery. Vol. 5, No. 2, 2017, pp. 151-156. doi: 10.11648/j.sd.20170502.20

Received: December 27, 2017; Accepted: May 4, 2017; Published: May 11, 2017

\begin{abstract}
In order to solve the inconvenience of pluggable power transmission, this paper attempts to design a non-contact transmission energy system.In this paper, the system device is divided into transmitter and receiver, and the study designed a special mechanical structure to solve the problem of coil offset. The computer simulation and practical experiments get the transmitting and receiving coils at different distances in the transmission power and efficiency, and the optimal transmission distance between the transmitting and receiving coils is obtained. Through a large number of related experiments done in the air, the experimental results further validate the accuracy of the simulation theory. When the inverter input voltage is $100 \mathrm{~V}$ and the system frequency is $98.7 \mathrm{kHz}$, the system transmission power up to $613 \mathrm{~W}$, which can provide a scientific theoretical basis and reference for further underwater wireless power transmission experiment.
\end{abstract}

Keywords: Non-contact, Electric Power Transmission, Coil

\section{一种中功率无线电能传输系统设备研究与实现}

李晓鹏 ${ }^{1,2}$, 袁学庆 ${ }^{2 *}$, 许凯林 ${ }^{2}$

'计算机与控制学院, 中国科学院大学, 北京, 中国

${ }^{2}$ 中国科学院沈阳自动化研究所, 机器人国家重点实验室, 沈阳, 中国

邮箱

1xpxueshanfeitian@163.com (李晓鹏), yuanxueqing@sia.cn（袁学庆）, xukailin@sia.cn（许凯林）

摘要：为解决可插拔设备电能传输方式所带来的不便, 本文试图设计一种非接触传输能量系统, 系统设备主要分为发 射端与接收端两个部分, 并利用特定的机械结构来解决线圈偏移问题。通过计算机仿真以及实验测试, 得到收发线圈 在不同距离下的传输功率与效率以及最优距离。通过在空气中大量的实验, 已经初步验证了仿真理论的准确性, 当变 频器输入电压为 $100 \mathrm{~V}$, 系统频率处在 $98.7 \mathrm{KHz}$ 时, 系统的传输效率可高达 $91 \%$, 系统传输功率可达 $613 \mathrm{~W}$, 可为进一步的 水下无线电能传输实验提供了科学的理论基础和参考依据。

关键词: 非接触, 电能传输, 线圈 


\section{1. 引言}

当前小功率无线电能传输已经得到广泛应用, 如手 机终端无线充电, 笔记本无线充电。一直以来, 由于半 导体器件的瓶颈, 加上其高压安全性欠佳, 使得大中功 率无线电能传输技术迟迟未投入应用。但值得庆幸的是 随着研究水平的提升和理论的不断完善, 尤其是磁场耦 合技术的迅猛发展，无线电能传输的应用领域得到了不 断的拓展。鉴于磁场耦合式无线电能传输方式的诸多优 点, 此种方式也成为大多数学者的研究重点 [1]。

磁场耦合式电能传输包含磁耦合感应式无线电能传 输 (Magnetically Coupled Inductive Wireless Power Transfer, MCI-WPT) 和磁耦合谐振式无线电能传输 ( Magnetically Coupled Resonant Wireless Power Transfer, MCR-WPT) 两种方式 [2]。应用磁耦合感应式 进行电能传输则需较近的传输距离, 一般在几毫米之内, 且其传输电能的效率随着距离的增大而呈现指数式的衰 减, 因而使其在无线电能传输领域中的应用范围变得极 为有限; 用磁耦合谐振式进行电能传输时, 线圈结构发 生变化, 可传输距离增加, 解决了实际应用中的结构难 题; 综上所述, MCR-WPT成为最佳的非接触电能传输方式。 MCR-WPT分为多种补偿方式, 较为常见的补偿方式有串串 补偿 (原边串联, 副边串联)、串并补偿 (原边串联, 副边并联）、并并补偿（原边并联, 副边并联）、并串 补偿 (原边并联, 副边串联)。

线圈设计是MCR-WPT中的关键环节。设计线圈需要涉 及到复杂的物理问题, 并且传输线圈和接收线圈设计方 式种类繁多。最新的四线圈结构模型包含激励线圈、发 射线圈、接收线圈和负载线圈; 三线圈结构模型包含发 射线圈、接收线圈、负载线圈; 普通双线圈结构模型中 包含发射线圈和接收线圈。三线圈和四线圈结构模型通 常适用于负载较大的情况下 [3], 其特点是结构较为复杂, 参数繁多。综合考虑系统成本和复杂度两个因素后, 此 处选取双线圈结构模型作为研究设计的最佳对象。

\section{2. 系统设计}

\section{1. 系统拓扑结构选取}

对于发射线圈（原边）, 串联补偿是最有效的补偿 方式, 其有两个优点: 第一, 有利于减小变频器逆变桥 两端电压, 即逆变桥只需要承受前级输入电压, 减小了 对开关管的损害 [4]; 第二, 有利于提高发射线圈的品质 因素。对于接收端（副边）, 采用串联补偿的主要优点 有两个: 第一, 接收线圈（副边）和补偿电容发生谐振 时, 其反射到原边的阻抗呈纯阻性, 原边发生谐振无需 改变补偿电容, 不必改动系统中的硬件结构; 第二, 负 载变化对输出电压产生的影响较小 [5-6], 输出电压较为 稳定, 因而选择副边的补偿方式也为串联补偿。最终, 本系统原边和副边的补偿方式均选择串联补偿, 在系统 完全谐振的情况下副边反射到原边的阻抗呈纯阻性, 增 强了系统输出电压的稳定性, 简化了实际设计的难度。
此外, 系统互感不应当过大, 负载阻值不应过小, 负载 不匹配或互感过大都会引起频率分裂效应 [7]。

\section{2. MCR-WPT模型分析}

经过以上论述确定了耦合整个系统的补偿方式, 其 互感电路模型如下图1所示。其中 $C_{P} 、 R_{P} 、 L_{P}$ 分别为原 边的谐振电容、等效阻抗和发射线圈的自感值; $C_{S} 、 R_{S}$ 、 $R_{L} 、 L_{S}$ 分别是副边的谐振电容、系统等效负载电阻、自 感以及负载, $U_{i}$ 和 $U_{0}$ 分别为原边的输入电压和副边负载 两端电压。

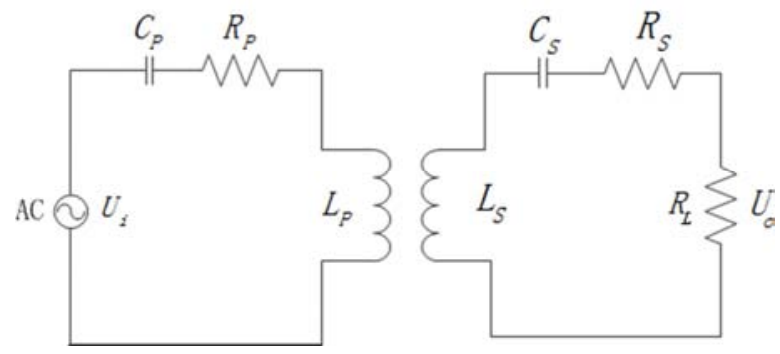

图1 互感电路。

对上述电路应用吉尔霍夫电压定律得到

$$
\left\{\begin{array}{l}
U_{i}=Z_{p} I_{p}-j \omega M I_{S} \\
U_{o}=j \omega M I_{p}-Z_{s} I_{s} \\
U_{o}=R_{L} I_{S}
\end{array}\right.
$$

当电容和电感完全相互补偿时有:

$$
\left\{\begin{array}{l}
j \omega L_{p}+\frac{1}{j \omega C_{p}}=0 \\
j \omega L_{s}+\frac{1}{j \omega C_{s}}=0
\end{array}\right.
$$

当整个系统满足条件 (2) 时, 系统的等效电路如下 图2所示，系统模型可以简化为式子（3）。

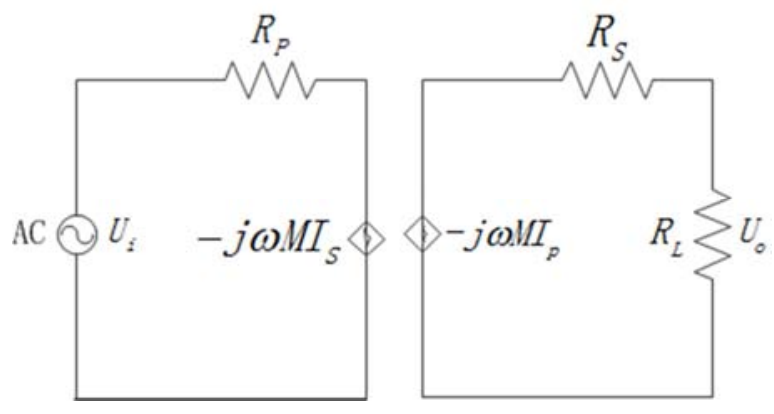

图2 等效互感电路。

$$
\left\{\begin{array}{l}
U_{i}=R_{p} I_{p}-j \omega M I_{S} \\
U_{o}=j \omega M I_{p}-R_{S} I_{s} \\
U_{o}=R_{L} I_{S}
\end{array}\right.
$$


对式子 (3) 进一步推导得系统的传输效率:

$$
\eta=\frac{P_{0}}{P_{i}}=\frac{\omega^{2} M^{2} R_{L}}{\left[R_{P}\left(R_{L}+R_{S}\right)+\omega^{2} M^{2}\right]\left(R_{L}+R_{S}\right)} \times 100 \%
$$

\section{3. 系统结构}

在实际操作中为了使线圈处于同轴且所处平面高度 平行, 需用特定的机械结构来满足这一要求, 从而保证 收发端线圈位置不发生偏移。设计时考虑用直线导轨与 滑块来满足这一实验环境要求, 其刚性高, 负荷能力强, 可调精度高, 实验装配示意如图3所示。通过导轨底座的 滑块调节，可实现收发端不同对接距离的调节与锁定。

在设计时, 需要考虑发射端与接收端绕线板材料为 非金属, 这样就避免了金属材料对无线充电的干扰。此 处线圈背板材料选择有机玻璃进行实验测试, 也可以为 P0M、PA6等常用非金属材料。

实验操作时, 发射端为固定端, 通过移动接收端调 整对接距离并按照距离由小到大的顺序进行测试, 记录 每种距离下对应的数据。

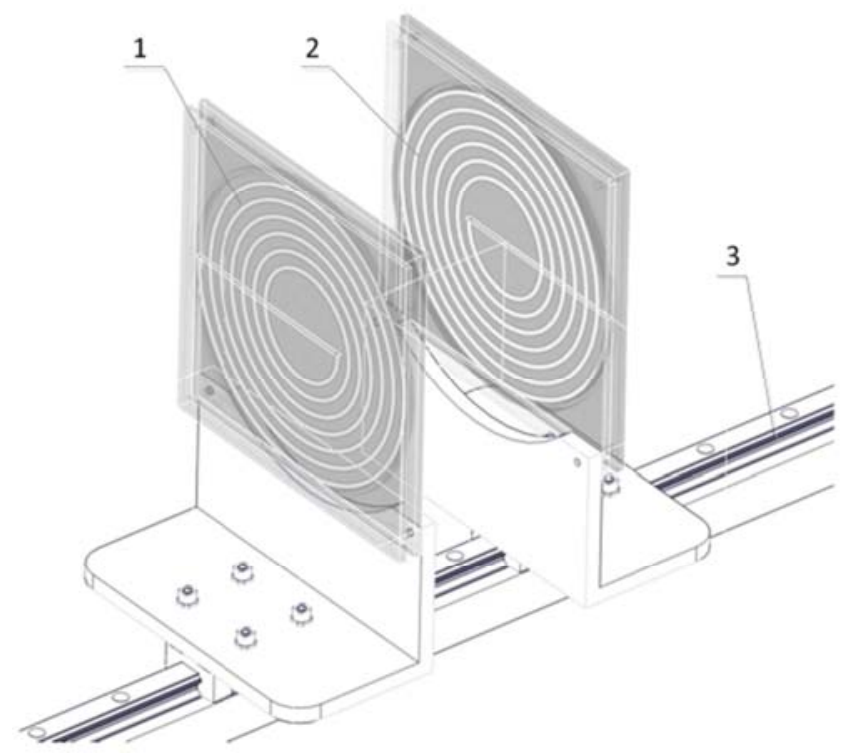

1. 发射端, 2. 接收端, 3. 直线导轨底座

图3 无线电能传输实验结构示意图。

\section{4. 线圈设计分析}

在系统原边和副边均选为串联补偿时, 现进一步对其 线圈进行设计。无线电能传输线圈形状也是人们当前研究 的最重要对象, 主流的线圈形状包括矩形线圈和圆形线圈。 而在大多数非接触能量传输中, 其线圈体积不应过大, 传 输功率和效率也要符合要求, 并且, 能量传输过程中一旦 确定了最佳线圈距离, 就不会对其进行改变。

由于设计中线圈采用了完全对称的结构, 即线圈的 中心轴都处在同一条直线上, 且两线圈所处平面完全平 行, 因此线圈没有发生相对偏移。鉴于圆形线圈结构简 单, 制作难度小, 设计时采用圆形线圈作为系统的收发 线圈, 其原理如图4所示。图中含有两个线圈模型, 左侧
为通用圆形线圈模型, 右侧模型为其特殊情况, 即两个 线圈处在同一条中心轴上。
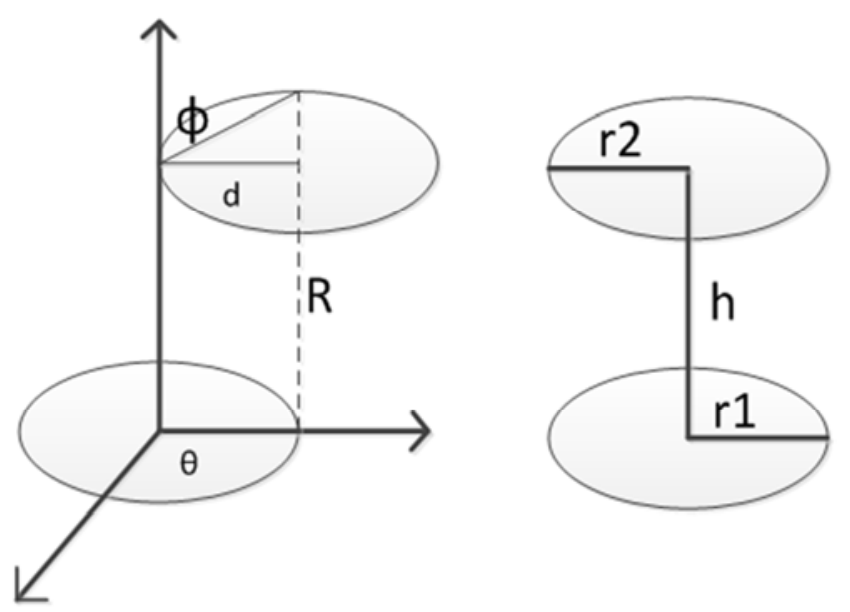

图4 圆形线圈原理示意图。

对上述模型应用诺伊曼公式得到互感数学模型（5）。

$$
M=\frac{N_{T} N_{R} \mu_{0}}{4 \pi} \iint_{L_{T} L_{R}} \frac{d 1_{T} d l_{R}}{R}
$$

式中, $\mathrm{R}$ 为两个线圈中微分对象之间的距离, $N_{T}$ 与 $N_{R}$ 为接收线圈和发射线圈的匝数, $\mu_{0}$ 为介质中的磁导 率。

将公式 (5) 化为极坐标形式, 可变换成公式 (6), 形式如下:

$$
M=\frac{N_{T} N_{R} \mu_{0}}{4 \pi} \int_{0}^{2 \pi} d \theta \int_{0}^{2 \pi} \frac{R_{1} R_{2} \cos \phi d \phi}{\left|R_{1}-R_{2}\right|}
$$

式中, $r_{1} 、 r_{2}$ 分别为发射线圈和接收线圈的线圈半 径, 其中有:

$$
\left\{\begin{array}{l}
R_{1}=r_{1} \cos \theta x+r_{2} \sin \theta y \\
R_{2}=r_{2} \cos (\theta+\phi)_{X}+\left[r_{2} \sin (\theta+\phi)+d\right]_{y}+h z
\end{array}\right.
$$

当上式中 $d=0$ 时, 即两线圈处于同一轴线时, 根据 [8] 的研究结果可得同轴线圈之间的互感近似计算约为:

$$
M \approx \frac{4.15 N_{T} N_{R} \mu_{0}}{2 R^{1,22}}(R+D)^{-2.22}[D \in(0.2 R, 10 R)]
$$

其中 $D$ 表示两同轴线圈间的距离，经过对公式 (8) 分析可知, 互感值变化与距离以及线圈半径密切相关。 当线圈半径确定时, 收发线圈间距越大, 其互感就越小。

趋肤效应是普通线圈的另一大特点, 趋肤效应会导 致感应线圈产生不良后果。使用普通的铜导线作为线圈 材料会导致线圈交流等效阻抗变大 [9-10], 线圈容易发 热, 传输效率降低, 久而久之会危害系统寿命。而盘式 绕线圈具有更高的品质因素 [11-12], 故此处采用专用的 利兹线来绕制线圈。实际实验中, 为方便计算, 在系统 
设计中制作了两个 $100 \mathrm{uH}$ 的盘式线圈分别作为发射线圈和 接受线圈，其实物图如下图5所示:

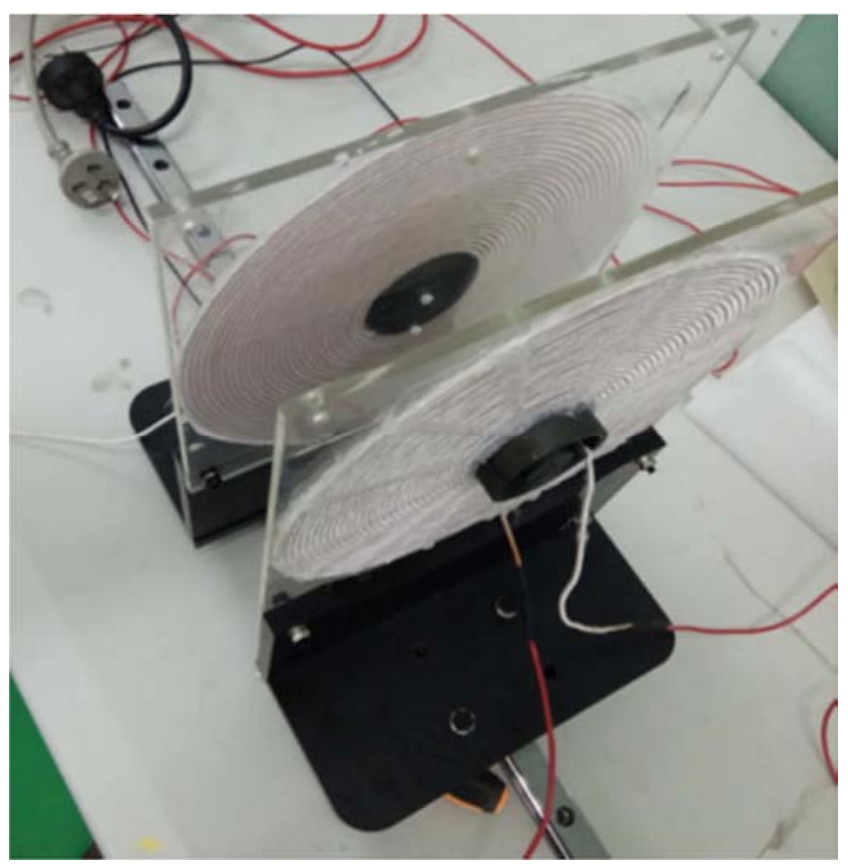

图5 线圈实物图。

\section{3. 仿真分析及实验结果验证}

\section{1 . 仿真分析}

对于无线电能传输设备而言, 传输功率与效率是设 计者最为关心的两个参数。根据公式 (2) 以及公式 (3) 推导出输出功率公式如下:

$$
P_{o}=I_{s}^{2} R_{L}=\frac{\omega^{2} M^{2} U_{i}^{2} R_{L}}{\left[R_{P}\left(R_{L}+R_{S}\right)+\omega^{2} M^{2}\right]^{2}}
$$

通过电桥等实验仪器, 测得系统各项参数, 如表1所 示。其中, $\omega$ 为系统频率, 并且, 该处忽略半导体器件 的压降, 设定变频器输入电压 $U_{i}$ 与发射电路两端电压 $U_{P}$ 相同。

表1 系统参数表。

\begin{tabular}{ll}
\hline 参数 & 数值 \\
\hline$C_{p}$ & $25.7 \mathrm{nF}$ \\
$C_{s}$ & $25.7 \mathrm{nF}$ \\
$R_{p}$ & $0.192 \Omega$ \\
$R_{S}$ & $0.19 \Omega$ \\
$L_{p}$ & $100 \mathrm{uH}$ \\
$L_{S}$ & $100 \mathrm{uH}$ \\
$\omega$ & $100 \mathrm{KHz}$ \\
$U_{i}$ & $50 \mathrm{~V}$ \\
$R_{L}$ & $20 \Omega$ \\
\hline
\end{tabular}

通过以上给定的参数, 以及公式 (4) 与公式 (9) 得到matlab仿真图如下所示。
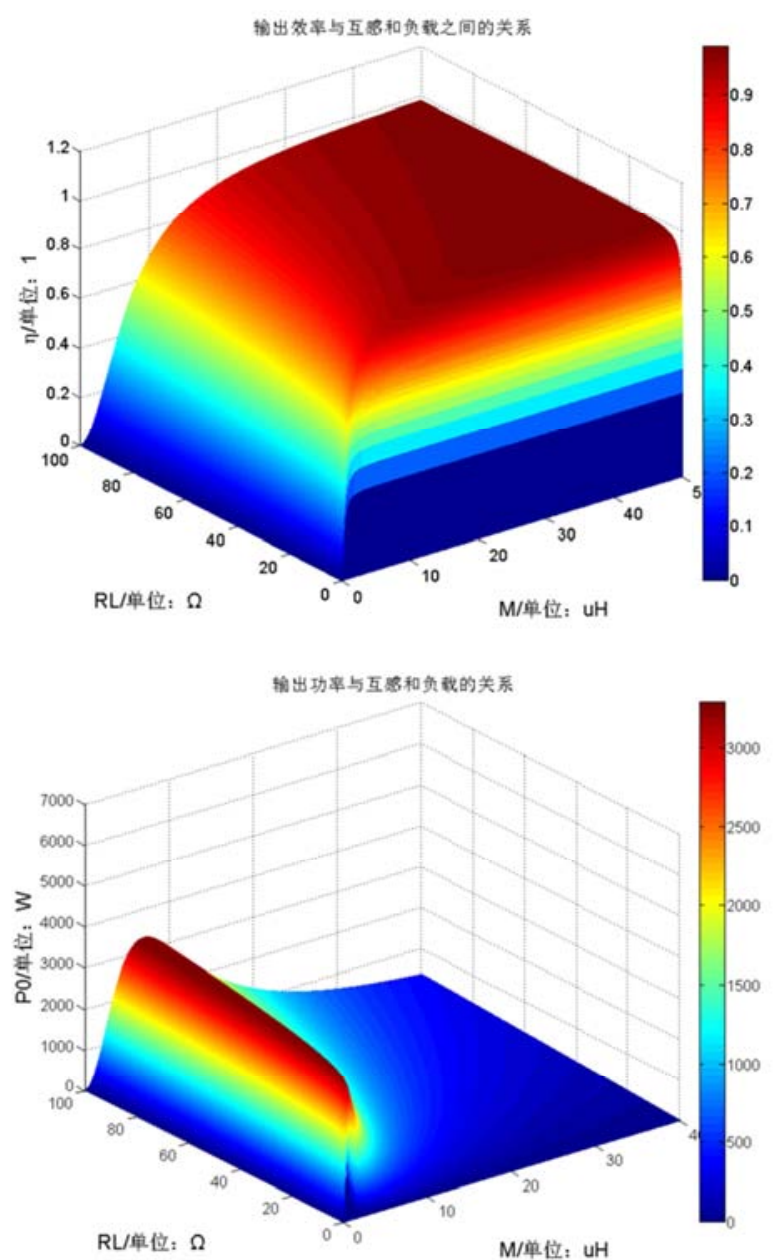

图6 Matlab仿真图。

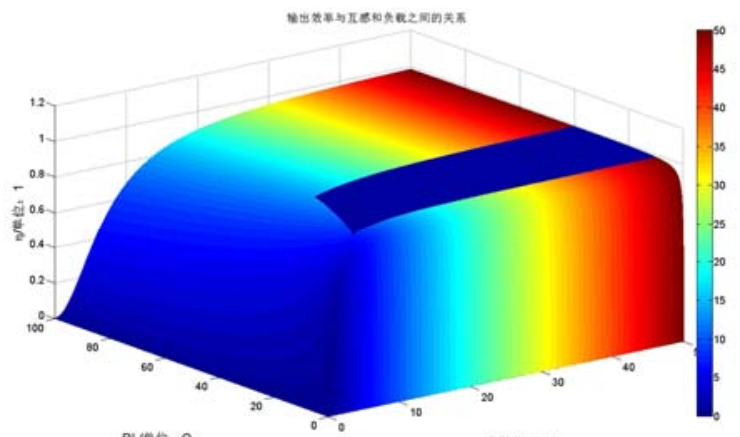

Muter, at

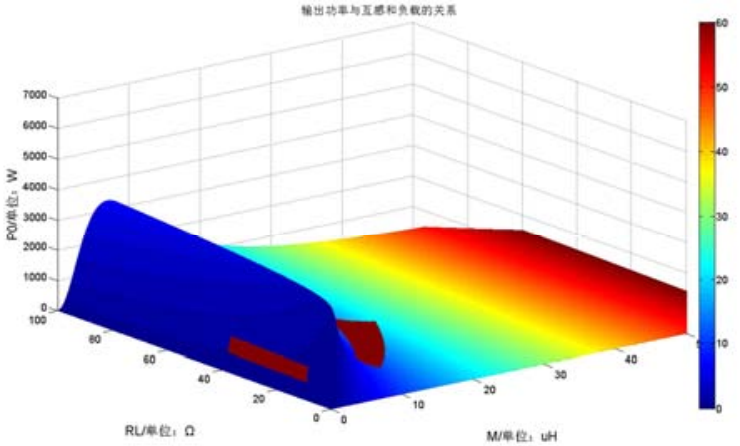

图7 Matlab含标定仿真图。 
通过对图6总体分析可得知, 其趋势是互感越大, 总 体效率就越高, 但系统输出功率会同步降低。因此, 在 实际设计过程需要选择合适的互感值, 使其既能满足功 率要求, 又能满足效率要求。在实际的实验中, 各种目 标参数和实际系统参数组成了系统约束, 通常情况下, 其包含了实际负载, 输出功率以及输出效率等要素, 而 本文中考虑了系统负载, 输出效率和输出功率, 实际系 统负载范围处于 $10-40 \Omega$; 系统功率要求输出功率在5001000 W之间; 系统效率则处在90\%-100\%之间。

通过对参数添加约束后, 得到含标定的仿真图 7 , 从 中可以看出, 当不考虑输出功率时, 其满足条件的部分 区域颜色为深蓝色, 而互感要需大于 $7 \mathrm{uH}$ 即可; 当只看输 出功率时, 其满足实际需求部分区域颜色为红棕色, 其 满足条件的互感范围分别是 0-3uH和 10-20uH。综上所述, 若要满足系统要求, $0-3 \mathrm{uH}$ 的互感范围不符合系统传输要 求, 因而可将理论互感选择区间设定为 $10-20 u H$ 。由于互 感越靠近 $20 \mathrm{uH}$, 就越有利于提高传输功率。因此, 可通 过直线导轨来设定最佳互感值, 也可进一步分析阻抗对 于与传输效率的影响。在实际应用中, 负载阻值范围一 般不超过 $50 \Omega$, 故只需分析负载在 $0-60 \Omega$ 的情况。在两 线圈互感值处于 $5-10 \mathrm{uH}$ 时, 电压增益比较大, 应尽量避 免在这个互感区域内接入较大的负载, 防止损坏负载端 半导体器件。

\section{2. 实验验证}

实验中使用了特定的机械装置来调节线圈间距, 实 验平台如图8所示。其主要构成为: $1.5 \mathrm{KW}$ 的直流电源, 最高频率为 $500 \mathrm{KHz}$ 的可调变频器, 采用高速二极管的整 流装置以及立兹线绕制的线圈, 立兹线横截面积为 $2.5 \mathrm{~mm}^{2}$, 发射线圈和接收线圈几何参数一致, 其内径和 外径分别为 $47 \mathrm{~mm}$ 和 $196 \mathrm{~mm}$ 。通过在线圈中心加入磁芯来增 大线圈的自感值, 使其保持在 $100 \mathrm{uH}$ 左右。此外, 磁芯还 能够改善磁场分布, 达到 “聚磁” 的效果。

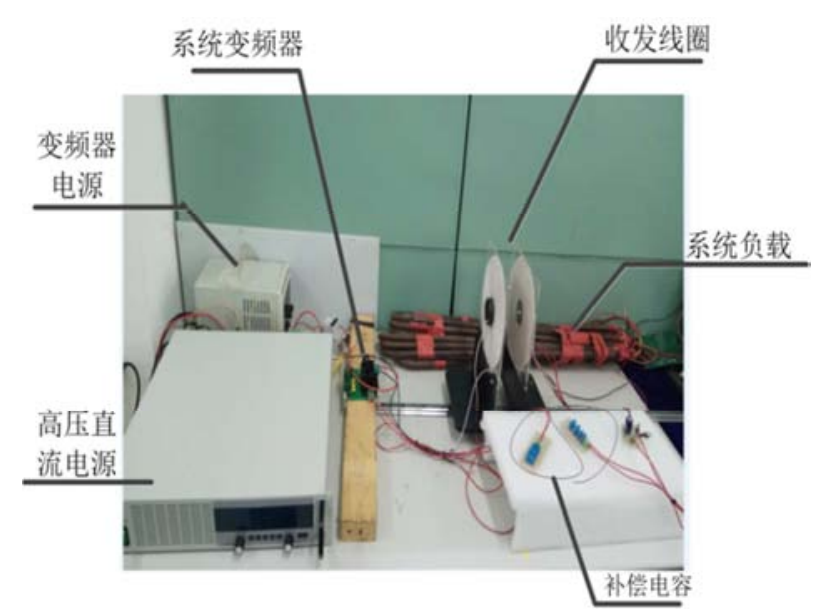

图8 无线充电实验平台。

在实际操作中, 较高的输入电压时会使电器元件发 热量增加, 副边整流管在高输入电压的情况下, 发热尤 为明显, 因此本实验设定输入电压为 $20 \mathrm{~V}$, 测试后得到实 验数据如下表所示。
表2 实验数据表。

\begin{tabular}{|c|c|c|c|c|c|c|c|}
\hline 参数 & 数值 & & & & & & \\
\hline$d(\mathrm{~mm})$ & 56 & 64 & 72 & 80 & 88 & 96 & 104 \\
\hline$U_{p}(v)$ & 20 & 20 & 20 & 20 & 20 & 20 & 20 \\
\hline$I_{p}(A)$ & 0.9 & 1.2 & 1.5 & 1.9 & 2.3 & 2.6 & 2.8 \\
\hline$U_{s}(v)$ & 18. 66 & 21.32 & 23.87 & 26. 07 & 27.68 & 28.52 & 28.39 \\
\hline $\bar{M}(u H)$ & 27 & 23 & 19.5 & 16.8 & 14.4 & 12.5 & 10.8 \\
\hline$P_{o}(w)$ & 16.98 & 22.17 & 27.79 & 33.15 & 37.37 & 39.67 & 39.31 \\
\hline
\end{tabular}

表2 继续。

\begin{tabular}{lllllll}
\hline 参数 & 数值 & \multicolumn{7}{l}{} \\
\hline$d(\mathrm{~mm})$ & 112 & 120 & 124 & 132 & 144 & 150 \\
$U_{p}(\mathrm{~V})$ & 20 & 20 & 20 & 20 & 20 & 20 \\
$I_{p}(A)$ & 2.9 & 2.8 & 2.8 & 2.7 & 2.5 & 2.4 \\
$U_{s}(\mathrm{~V})$ & 27.6 & 26.0 & 25.1 & 23.4 & 20.4 & 18.9 \\
$\bar{M}(u H)$ & 9.4 & 8.2 & 7.75 & 6.27 & 5.75 & 5.27 \\
$P_{o}(W)$ & 37.2 & 33.5 & 30.9 & 26.71 & 20.38 & 17.51 \\
\hline
\end{tabular}

上述表格中 $d 、 U_{P} 、 I_{P} 、 U_{S} 、 \bar{M} 、 P_{0}$ 分别表示线圈 间距、变频器输入电压、变频器输入电流、副边整流管 输出电压、互感值、输出功率, 其中输出功率 $P_{o}=U_{s}^{2} / R_{L}$ 。因系统频率不高, 故计算中可忽略器件导 通电阻。将实验数据绘制成直观的图像曲线如下图9所示。
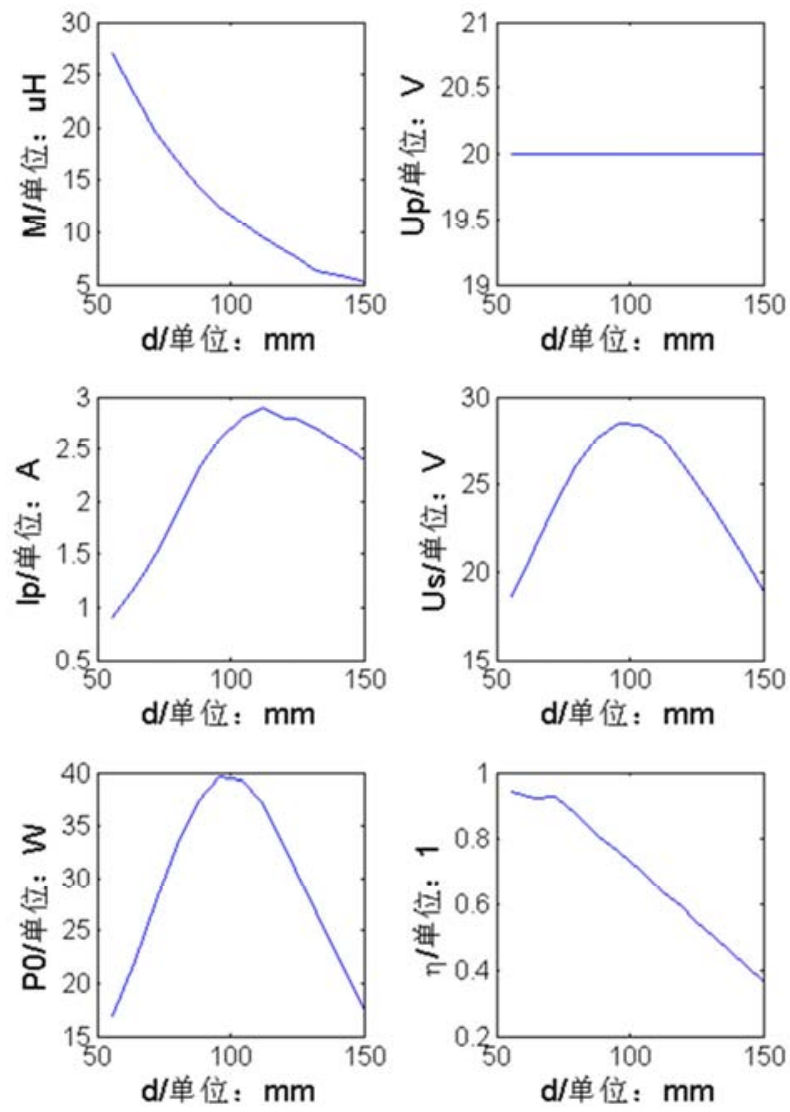

图9 实验曲线。 
由上述曲线可知, 当收发线圈的距离增加时, 其互 感值呈现非线性下降。当设定原边电压、负载值以及系 统频率保持不变时, 原边电流随着距离的增大呈现先增 大后减小的趋势, 输出功率以及副边电压都呈现先上升 后下降的趋势, 而传输数效率随着距离的增大而下降。 通过实验标定, 要使传输效率大于 0.9 时, 需要保证收发 端距离在50-85 mm的区间内; 而使输出功率达到较高时, 需保证的收发端距离范围是 65-110mm。综上所述, 实验 中标定选取的最佳距离为 $72 \mathrm{~mm}$, 此时, 传输效率可达到 $93.9 \%$ 。当系统互感系数约为 $19 \mathrm{uH}$ 时, 在 $20 \mathrm{~V}$ 电压输入下, 输出功率为 $28.45 \mathrm{~W}$; 当输入电压为 $50 \mathrm{~V}$ 时, 输出功率为 $182 \mathrm{~W}$; 当输入电压为 $80 \mathrm{~V}$ 时, 输出功率为 $457 \mathrm{~W}$; 当输入电 压为 $100 \mathrm{~V}$ 时, 输出功率可达 $613 \mathrm{~W}$ 。为减小系统装置体积, 线圈面积应当尽量小, 线圈距离也不应过大。综上所述, 收发端线圈距离保持在 50-120mm内为最佳。

\section{4. 结论}

本文设计了一种面向无线电能能量传输的非接触系 统, 文中主要对电路原理进行了分析, 并简要论述了线 圈设计的基本要点, 同时对电路的数学模型进行了实验 仿真以及初步实验验证。通过由仿真分析选取的有效参 数进行的实验表明, 当负载在 $20 \Omega$ 、接收端和发射端的 互感值为 $20 \mathrm{uH}$ 时, 系统既可以满足一定的输出功率, 也 可以满足一定的输出效率。在实验中, 系统输出效率始 终保持在 $90 \%$ 以上，很好的证明了仿真模型的准确性和原 始设计的有效性, 为进一步验证水下无线电能传输提供 了重要的参考依据。

\section{参考文献}

[1] 李素环, 廖承林, 王丽芳, 等. 无线电能传输中线圈设计 对效率的影响综述 $[J]$. 电工技术学报, 2015, 30 (1) : 270275 。

［2］张克涵, 阎龙斌, 阎争超, 等. 基于磁共振水下非接触式 电能传输系统建模与损耗分析 $[\mathrm{J}]$. 物理学报, 2016, 65 (4):048401 (1-9)。
[3] 王国东, 乔振鹏, 王允建等. 磁耦合谐振式无线电能传输 系统中线圈谐振特性研究 $[\mathrm{J}]$. 电源学报, 2015, 13 (2) :58-63。

[4] 毛赛君. 非接触感应电能传输关键技术 [D]. 南京航空航 天大学, 2006。

[5] 施松. 电动车无线供电系统拾取装置的设计 [D]. 重庆大 学, 2013。

[6] J. M. Miller, 0. C. Onar, and M. Chinthavali, "Primary-side power flow control of wireless power transfer for electric vehicle charging," IEEE Journal of Emerging and Selected Topics in Power Electronics, vol. 3, no. 1, pp. 147-162, March 2015.

[7] A. P. Sample, D. A. Meyer, and J. R. Smith, "Analysis, experimental results, and range adaptation of magnetically coupled resonators for wireless power transfer, " IEEE Trans. Ind. Electron., vol. 58, no. 2, pp. 544-554, Feb. 2011.

[8] 会张伟, 陈竣斌. 同轴等大线圈互感系数及相互作用力的 近似解析公式 $[J]$ 。大学物理, 2004, 23(8):36-40。

[9] 卢闻州, 沈锦飞, 方楚良. 磁耦合谐振式无线电能传输电 动汽车充电研究 $[J]$. 电机与控制学报, 2016, 20 (9) : 4653 。

[10] 卢秋朋, 张清鹏, 秦润杰. 传输线中趋肤效应的介绍及仿 真 $[J]$. 电子测量技术, 2015, 38 (6) :27-30。

[11] 张献, 杨庆新, 崔玉龙等. 大功率无线电能传输系统能量 发射线圈设计、优化与验证 $[\mathrm{J}]$. 电工技术学报, 2013, 28 (10) : 12-18。

[12] S. Cheon, Y. Kim, S. Kang, M. Lee and T. Zyung. : Circuit-model-based analysis of a wireless energytransfer system via coupled magnetic resonances, IEEE Transactions on Industrial Electronics vol. 58, pp. 2906-2914, 2011. 